\title{
Wikis - Bestandsaufnahme eines Forschungsfeldes und Skizzierung eines integrativen Analyserahmens
}

\author{
Christian Pentzold / Sebastian Seidenglanz / Claudia Fraas / Peter Ohler
}

Wikis als social software und Form netzbasierten kollaborativen Arbeitens markieren einen entscheidenden Schritt in der jüngeren Geschichte des Webs. In Funktionsumfang und Popularität lassen sie bereits jetzt ältere Generationen von Webangeboten hinter sich und ebnen den Weg für neue Formen virtueller Interaktion. Der vorliegende Beitrag erschließt dieses für die Kommunikations- und Medienwissenschaften fruchtbringende Forschungsfeld, indem er einen Überblick über die bisherige Wiki-Forschung, insbesondere im Blick auf die Online-Enzyklopädie Wikipedia, gibt. Darüber hinaus skizziert er die Ansätze eines integrativen Analyserabmens, der die Online-Enzyklopädie Wikipedia aus einer medienlinguistischen und mediensoziologischen Sicht als soziales System konzeptualisiert. Dabei werden zum Stand der Forschung strukturierungstheoretische und genreklassifikatorische, handlungs-und konsensorientierte sowie informationstechnische Ansätze ebenso dargestellt wie Arbeiten zur Informationsqualität, zu Nutzerklassifikationen und Motivlagen.

Schlagwörter: Wiki, Wikipedia, Social Software, Computervermittelte Kommunikation, Online-Kollaboration, Online-Vergesellschaftung, Wissensgenerierung

\section{Einleitung ${ }^{1}$}

Wikis als kollaborative Hypertext-Systeme zählen zu den erfolgreichsten Entwicklungen der jüngeren Geschichte des Web. Zusammen mit Weblogs ${ }^{2}$ und anderen Formen von user generated content ${ }^{3}$ gehören sie zu einer neuen Generation webbasierter Angebote, welche Foren, Chatrooms und Newsgroups in ihrem Funktionsumfang und ihrer Popularität allmählich hinter sich lassen. So gehört etwa das bislang größte Wiki, die Online-Enzyklopädie Wikipedia, zu den 20 derzeit am häufigsten besuchten Webseiten. Im letzten Quartal 2006 wurde sie im Schnitt täglich mehr als 40 Millionen mal angeklickt (vgl. das Ranking auf alexa.com).

Ein neues Forschungsfeld eröffnet sich, dessen Analyse aus kommunikations- und medienwissenschaftlicher Sicht fruchtbringend scheint. Daher sollen im vorliegenden Beitrag zum einen die zentralen Befunde der bislang vorliegenden Arbeiten beschrieben und systematisiert, zum anderen ein integrativer Analyserahmen skizziert werden, welcher Wikipedia aus einer medienlinguistischen und -soziologischen Sicht als soziales System konzeptualisiert. Der Forschungsstand zu Wikis, insbesondere zur Wikipedia, wird unter folgenden Gesichtspunkten dargestellt: 1. strukturierungstheoretische und genreklassifikatorische Ansätze, 2. handlungs- und konsensorientierte Ansätze, 3. Ar-

1 Wir danken Sigmar Papendick, Stefan Meier und Thomas Möckel für ihre Unterstützung bei der Erstellung dieses Aufsatzes.

2 Weblogs sind ,Logbücher' im Netz, die periodisch neue Einträge erhalten, wobei die neuesten an oberster Stelle stehen. Oftmals wird die Kurzbezeichnung Blog verwendet. Vgl. Fraas, 2005.

3 Dazu zählen z. B. Plattformen, die der Veröffentlichung von Videos (YouTube), Bildern (Flickr) und anderen Inhalten (z. B. MySpace) dienen. 
beiten zu Nutzerklassifikationen und Motivlagen sowie 4. Visualisierungstechniken und weitere informationstechnische Ansätze. Zunächst jedoch scheint ein Blick auf die prinzipielle Funktionsweise von Wikis und ihre Verortung im Bereich der social software angebracht.

\section{Funktionen und Eigenschaften von Wikis}

Seit ihrer erstmaligen Anwendung 1995 durch Ward Cunningham verbreiten sich Wikis zügig in sehr unterschiedlichen Kontexten und werden inzwischen in wirtschaftlichen (vgl. Andersen, 2004; Fuchs-Kittowski/Köhler, 2005), universitär-wissenschaftlichen (vgl. Augar/Raitman/Zhou, 2004; Bruns/Humphreys, 2005; Forte/Bruckman, 2006), schulischen (vgl. Désilets/Paquet/Vinson, 2005; Döbeli Honegger, 2005; Lund/Smødal, 2006) und zivilgesellschaftlichen Organisationen (vgl. Groß/Hülsbusch, 2004; Voß/Danowski, 2004; Frumkin, 2005) angewendet (vgl. Möller, 2005; Leitner, 2003). Bereits 2004 sprechen Chawner/Lewis von mehr als 1000 öffentlich zugänglichen Wikis. Damit sind sie eine prominente Form der social software.

\subsection{Wikis als social software}

Der Begriff social software wird in der neueren Diskussion häufig zur Eingrenzung eines Feldes verschiedener netzbasierter Anwendungen zum Informations-, Identitätsund Beziehungsmanagement gebraucht (vgl. Davies, 2003). Im Gegensatz zu Formen projekt- und organisationsorientierter Software, wie passwortgeschützten Plattformen oder Intranets, wird dabei der Bottom-up-Prozess der Community-Formation in den Vordergrund gestellt (vgl. Boyd, 2003). Social software umfasst Anwendungen, in denen die Nutzer miteinander kommunizieren und gegebenenfalls kollaborativ arbeiten können, die prinzipiell für jeden zugänglich sind, kaum ausdifferenzierte Nutzerhierarchien aufweisen und die Möglichkeit der freien Kombination von One-to-one, One-to-many und Many-to-many-Kommunikation bieten. Anders als die aus dem Bereich der Opensource-Bewegung bekannten Communities, in denen meist Experten organisiert sind, stellt social software ein niedrigschwelliges Angebot dar (vgl. Schmidt, 2006: 174f.).

Das Spektrum der Formen reicht von bereits klassischen Online-Diensten wie Foren und Newsgroups über Messenger Systeme bis hin zu Weblogs, Social-NetworkingSeiten und Wikis (vgl. Shirky, 2003; 2003a). Ihre Entwicklung kann unter einer techniksoziologischen Perspektive hierbei als dynamischer Vorgang der kontinuierlichen Anpassung und Veränderung beschrieben werden, d. h., ein Teil der Software selbst entsteht in sozialen Prozessen, sie wird fortwährend den Nutzerbedürfnissen entsprechend adaptiert und weiterentwickelt (vgl. als Überblick Lievrouw, 2002). Statt langer Entwicklungszyklen und großer Versionssprünge dominiert eine Form fortlaufender Programmierung.

\subsection{Das Wiki-Konzept}

Grundlegendes Prinzip der Wikis (von wikiwiki, hawaiianisch für schnell) ist die vollständige Offenheit der Inhalte, d. h., jeder Benutzer kann diese erstellen, ändern und gegebenenfalls auch löschen. Damit stellen sie ein kollaboratives Open-content-System dar, das im Kontext von bereits existierenden Clients, Protokollen und Standards arbeitet (vgl. Leuf/Cunningham, 2001:12) und im Bereich der Diskussions- und Kollaborationsserver (vgl. ebd.: 3) eingeordnet werden kann (vgl. zur technischen Umsetzung Ebers- 
bach/Glaser/Heigl, 2005; Lange, 2005). Wikis sind offene, geteilte Wissensspeicher (vgl. Paquet, 2003: 56), die Diskussionsräume eröffnen, in denen Wissen (re-)produziert und organisiert wird. Damit nutzen sie konsequent das Interaktionspotenzial des Webs. ${ }^{4}$

Ausgangspunkt für die Entwicklung des Wiki-Konzeptes war laut Cunningham seine Unzufriedenheit mit den gängigen Textverarbeitungsprogrammen, die nur bedingt ein gemeinschaftliches Arbeiten an Dokumenten erlaubten. Das Portland Pattern Repository (PPR) als erstes Wiki - „the simplest online database that could possibly work“ (Leuf/Cunningham, 2001: 15) - hingegen ermöglicht es den Autoren, die Inhalte synchron oder asynchron zu bearbeiten. Darüber hinaus dokumentiert die Software jeden Bearbeitungsschritt in Artikelhistorien, während die aktuelle Version jeweils als Volltext erscheint. Die Editiermöglichkeiten umfassen im Wesentlichen Textänderungen, -hinzufügungen, -löschungen und -verlinkungen. Die Inhalte und die unterschiedlichen Wikisoftwarelösungen selbst unterliegen oftmals der GNU General Public License und der GNU Free Documentation License (GPL/FDL), d. h., sie können daher frei genutzt, verbreitet und bearbeitet werden (vgl. GPL, 1991 und FDL, 2002). ${ }^{5}$

\subsection{Wikipedia - Entwicklung, Funktionen und Eigenschaften}

Das Projekt „Wikipedia“ stellt bislang das mit Abstand größte Wiki dar. Die kollaborative Enzyklopädie wurde im Januar 2001 von Jimmy Wales ins Leben gerufen. In den bisher vergangenen sechs Jahren hat sie sich zum größten Allgemeinlexikon der Welt entwickelt. ${ }^{6}$ Die englischsprachige Version umfasst derzeit mehr als 1.583 .500 angelegte Artikel und rund 3.254.800 angemeldete Benutzer, die deutsche als zweitgrößte über 527.700 Artikel. Im Januar 2007 waren über 336.500 Benutzer mit einem Benutzernamen registriert, wobei die Zahl der Autoren höher liegen kann, da Beiträge auch anonym erstellt werden können. In diesem Fall erscheint statt des Nutzernamens die jeweilige IP-Adresse (Stand: 15. Januar 2007; vgl. Wikipedia:Special:Statistics bzw. Wikipedia: Spezial:Statistics). Im Moment verdoppelt sich der Inhalt der englischsprachigen Wikipedia jedes Jahr. Das Wachstum der deutschen Version verlief Voß (2005) zufolge bisher in drei aufeinanderfolgenden Phasen: linear (03/2001-03/2002), exponentiell (04/2002-02/2005) und wieder linear (ab 03/2005). Die Artikelzahl steigt um ca. 10.000 pro Monat. Ein ähnlicher Verlauf ist nur noch für die japanische Wikipedia feststellbar, während in anderen Ausgaben (insgesamt 229 Sprachen) teilweise sogar eine Verdreifachung des Inhalts innerhalb eines Jahres stattfindet. Eine andere Entwicklung nimmt hingegen das Wachstum der angemeldeten User. Hier wird deutlich, dass die Anzahl der hinzukommenden Nutzer ansteigt, die Zahl der aktiven Autoren jedoch ständig fluktuiert (vgl. Zachte, 2006).

Herausragend ist insbesondere die außerordentliche Leistungsfähigkeit, hohe Stabilität und komplexe Organisation der Wikipedia, welche erstaunlicherweise im Grunde

4 Vgl. auch Aronsson (2002: 27), der von einem Wiki als „hypertext on steroids“ spricht. Neben dem vollständig freien Zugang sind aber auch Modelle der Zugangsbeschränkung denkbar. Vgl. Leuf/Cunningham (2001: 277).

5 Die GPL und die FDL gehen auf eine Idee Richard Stallmans zurück. GNU bezeichnet dabei das von Stallman vorangebrachte Projekt zur Bereitstellung eines freien Betriebssystems. Die Lizenzen erschließen vier grundlegende Nutzungsmöglichkeiten: die uneingeschränkte Nutzung, die Verbreitung von Kopien, das Studieren der Funktionsweisen und die Bearbeitung dieser. Die Nutzungsformen gelten gemäß der FDL auch für die Inhalte der Wikipedia.

6 Zum Vergleich: Die Encyclopeedia Britannica bietet in 32 Bänden 75.000 Artikel mit ca. 44 Mio. Wörtern, der Brockhaus in 30 Bänden mehr als 300.000 Artikel mit ca. 33 Mio. Wörtern. 
auf nur vier fundamentalen und explizit formulierten Regeln beruht: 1. „Wikipedia ist eine Enzyklopädie.“ 2. „Neutralität des Standpunktes.“ 3. „Keine Verletzung des Urheberrechts.“ 4. „Keine persönlichen Angriffe.“ (Wikipedia:Richtlinien). Ein großer Teil der Artikel erreicht trotz der kompletten Offenheit auch gegenüber Vandalismus ein hohes Maß an Vollständigkeit und Richtigkeit. ${ }^{7}$ So wurden vom Magazin Nature zum Vergleich 42 zufällig gewählte Beiträge aus der Wikipedia und der Encyclopcdia Britannica ohne Angabe der Quelle verschiedenen Wissenschaftlern vorgelegt (vgl. Giles, 2005). In der Untersuchung konnten nur acht schwere Fehler in Wikipedia-Artikeln identifiziert werden. Insgesamt hatten die untersuchten Beiträge 162 faktische Fehler, Auslassungen bzw. Irreführungen, die Artikel der Encyclopedia Britannica jedoch ebenfalls 123 (vgl. auch die ähnlichen Ergebnisse bei Rosenzweig, 2006).

Der wesentliche Grund für die Leistungsfähigkeit, d. h. für das enorme Wachstum der Wikipedia bei gleichzeitig zumeist hoher Qualität der Informationen, scheint in der Wiki-Idee selbst zu liegen. Durch die oben beschriebene völlige Offenheit der Texte, die auch ohne vorherige Anmeldung bearbeitet werden können, wird eine sehr niedrige Schwelle für potenzielle Autoren gesetzt. Ein Blick in die Geschichte der Wikipedia zeigt, wie wesentlich dieser Schritt hin zur radikalen Freigabe der Inhalte war. Ihr Vorläufer, das Online-Lexikon Nupedia, war im März 2000 als Peer-review-Projekt unter Leitung eines bezahlten Herausgebers, Larry Sanger, gegründet worden. Die Arbeitsweise sollte dergestalt funktionieren, dass sich jeder Webuser anmelden und an der Artikelproduktion sowie dem Review-Prozess teilnehmen konnte. Die Schwelle zum Eintritt war aber mit der Erwartung „We wish editors to be true experts in their fields and (with few exceptions) possess Ph.D.s.“ (Nupedia, 2000) sehr hoch gesetzt, so dass die Zahl der angemeldeten Nutzer nicht über 2000 hinausstieg. Außerdem gelang es innerhalb eines knappen Jahres nur, ca. 24 als fertig angesehene Artikel zu produzieren (vgl. Sanger, 2005). Erst durch die Abschaffung des Review-Verfahrens und der Anmeldepflicht wurde es möglich, die Enzyklopädie für viele User attraktiv zu machen. Die Zahl der Autoren stieg daraufhin sprunghaft an, und eine den Open-source-Projekten vergleichbare Dynamik setzte ein (vgl. Garcia/Steinmueller, 2003). Die oftmals hohe Qualität der Inhalte wiederum scheint ebenfalls mit der Größe der Autorenzahl in Verbindung zu stehen, gemäß dem Slogan von Eric Raymond, dem Vordenker der Opensource-Bewegung, „many eyes make all bugs shallow“ (Raymond, 2000). Erleichtert wird die Zusammenarbeit darüber hinaus durch die in der Wikipedia-Software (das spezielle Programm heißt „MediaWiki“) angelegte Transparenz des Editierprozesses (vgl. Abb. 1). Erzielt wird sie vor allem durch die Dokumentationsfunktion, denn jede Änderung wird automatisch als Editierversion mit Angabe der Änderungszeit, dem jeweiligen Editor und dessen Kommentar abgespeichert (vgl. auch Fiebig, 2005; Danowski/Voß, 2005; Miller, 2005; Schliecker/Lehmann, 2005; Wirth/Brändle, 2006).

In den Kommentaren selbst ist im Laufe der Zeit eine Form wikipediaspezifischer Sprache (z. B. Abkürzungen, Schlüsselworte) entstanden. Die kurzen Beiträge der Autoren werden häufig zur Erörterung von begrifflichen oder thematischen Unklarheiten genutzt, wobei die Diskussionen in den jeweiligen Diskussionsseiten der Artikel aufgenommen werden können, wenn sich z. B. Konflikte zu edit wars, d. h. längeren Auseinandersetzungen, entwickeln. ${ }^{8}$ Die Diskussionsseiten bilden damit ein wichtiges

7 Zur möglichen Definition von Vandalismus vgl. die im Absatz 3.4 referierte Gliederung von Viégas/Wattenberg/Dave, 2004.

8 Die Verbindung aus Artikel, Versionsgeschichte und Diskussionsseiten bezeichnen Kohl/Liebert (2004: 139) als Textverbund. 
Abbildung 1: Die Versionshistorie des Artikels „Angela Merkel“

\begin{tabular}{|c|c|c|}
\hline & Dislcussion & Versionen/Autoren \\
\hline 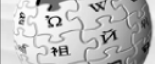 & \multicolumn{2}{|r|}{ Die Kandidatur fur die Wiahl des Kulatoriums der UVisemedia Foundation lautt. } \\
\hline WIIKIPEDIA & \multicolumn{2}{|c|}{$\begin{array}{l}\text { Frühere Versionen } \\
\text { Logioucher tür diese Seïe anzeigen } \\
\text { (Neueste | Älteste) Zeige (vorherige 50) (nảchste 50) (20 | } 50 \text { | } 100 \mid 250 \text { | 500) }\end{array}$} \\
\hline $\begin{array}{l}\text { Navigation } \\
\text { - Hauptsefte } \\
\text { - Uber Wikipedia } \\
\text { - Themenportale } \\
\text { - Van A bis Z }\end{array}$ & \multicolumn{2}{|c|}{ 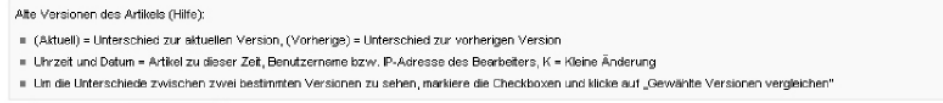 } \\
\hline - Zufälliger Artikel & \multicolumn{2}{|c|}{ Gewrählte Versionen vergleichen } \\
\hline Mtmachen & \multicolumn{2}{|c|}{$\begin{array}{l}\text { - (Aktuell) Norherige) } \odot \text { 12:19, 16. Aug } 2006 \text { Abe Lincoln (Diskussion I Beitrage) ( } \rightarrow \text { Bisherige Amtszeit - update, siehe } \\
\text { http://wnw spiegei de/politil/deutschland } 0,1518,431916,00 \text { html) }\end{array}$} \\
\hline $\begin{array}{l}\text { - Hilfe } \\
\text { - Whipedia-Portal } \\
\text { - Letzte Anderungen } \\
\text { - Spenden }\end{array}$ & $\begin{array}{l}\text { - (Aktuell) (Vorherige) } \odot \\
\text { wissenschaftiche arbed) }\end{array}$ & $\begin{array}{l}\text { (itikdeutachland 0, 1618,43916,00 html) } \\
\text { 07:00, 14. Aug } 2006 \text { Abe Lincoln (Diskussion | Eeiträge) (revert, das steht bereils am ende des abschnits }\end{array}$ \\
\hline Antikel Suche & \multirow{2}{*}{$\begin{array}{l}\text { - (Aktuel) (Vorherige) } 0 \\
\text { - (Aktuel) (Vorherige) } 0 \\
\text { - (Aktuell) (Vorherige) } 0 \\
\text { - (Aktuel) (Vorherige) } 0 \\
\text { - (Aktuell) (Vorherige) } 0 \\
\text { - (Aktuell) (Vorherige) } 0 \\
\text { - (Aktuell) (Vorherige) } 0 \\
\text { - (Aktuell) (Vorherige) } 0 \\
\text { - (Aktuell) (Vorherige) } 0 \\
\text { - (Aktuell) (Vorherige) } 0\end{array}$} & $\begin{array}{l}\text { 02:09, 14. Aug } 2006 \text { Robocop1 (Diskussion | Beitrăge) } \\
\text { 14:59, 12. Aug } 2006 \text { Dcon (Diskussion | Beitrảge) K (ah ok) } \\
\text { 14:46, 12. Aug } 2006 \text { Dcon (Diskussion I Beitrảge) (so } 40.000 \text { und wieso pov, wo soll der sein?) }\end{array}$ \\
\hline $\begin{array}{l}\text { Werkzouge } \\
\text { - Links aut dese Seite } \\
\text { = Ärndenungen an } \\
\text { verlinkten Seten } \\
\text { = rss atom } \\
\text { = Hochladen } \\
\text { - Spezialseten }\end{array}$ & & 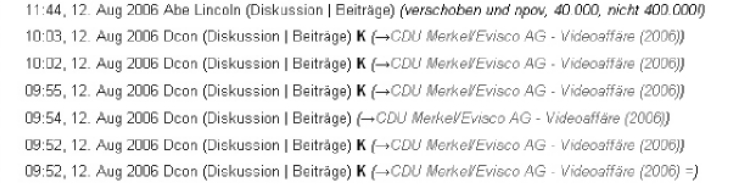 \\
\hline
\end{tabular}

Zuerst erscheint das Änderungsdatum, gefolgt vom Editor und dem Kommentar. Des Weiteren besteht die Möglichkeit, zwei Versionen auf die vorgenommenen Veränderungen hin zu vergleichen.

Instrument der Meinungsbildung im Entstehungsprozess des Artikels. In der deutschen Version werden ca. 19 \% aller potenziell verfügbaren, weil bei der Anlegung eines Lemmas ${ }^{9}$ automatisch erzeugten Seiten für die Diskussion genutzt. Dies entspricht rund 11,3\% der Gesamtdatenmenge der deutschen Ausgabe (insgesamt ca. 1.424.000 Seiten in der Datenbank). Damit besitzt sie im Vergleich mit anderssprachigen Ausgaben den größten Anteil an Diskussionsseiten. In der kroatischen sind es bspw. nur 2,4 \% aller Seiten und in der japanischen 9,8\% (vgl. Voß, 2005). ${ }^{10}$ Die deutsche Wikipedia besteht außerdem zu 59,1\% aus Lexikonartikeln, der Rest des Datenvolumens wird von Metadokumenten eingenommen. Diese Metadokumente wiederum existieren weitestgehend losgelöst von den Artikeln und stellen u. a. die Vereinbarung und Einhaltung von Normen sicher, helfen Formen von Vandalismus zu bekämpfen, dienen der Bestimmung von Administratoren oder exzellenten Artikeln, zur Organisation der Autoren oder sie geben Gelegenheit, allgemeine Themen zu diskutieren. Ein häufig genutztes Entscheidungsmittel sind die Meinungsbilder. Dabei werden Fragen, etwa zur (Un-)Rechtmäßigkeit eines Löschantrages für einen Artikel, mit einer zeitlichen Begrenzung zur Begutachtung freigegeben. Die Nutzer können ihre positiven/negativen Voten abgeben und somit über den Verbleib bzw. die Entfernung entscheiden (vgl. die umfangreiche Dokumentation unter Wikipedia:Portal).

9 Wie in der Lexikografie und Linguistik üblich, wird auch in der Wikipedia der Begriff ,Lemma“ verwendet, um auf ein Stichwort in seiner Grundform zu verweisen.

10 Vgl. auch Hepp (2006), der darauf hinweist, dass Wikipedia prinzipiell global verfügbar ist, sich aber als Enzyklopädie entlang von Sprachbarrieren begrenzter Kommunikationsräume konstituiert. 


\section{Forschungsstand zu Wikis/Wikipedia}

Nunmehr sollen die verstreuten Ansätze einer wissenschaftlichen Beschäftigung mit Wikis und insbesondere mit Wikipedia überblicksartig dargestellt werden. Dabei sei vorangestellt, dass diese meist auf einer deskriptiven Stufe stehen bleiben und Erklärungen über Eigenschaften und Arbeitsweisen dieser netzbasierten Kollaborationsform vermissen lassen. Vielmehr tragen sie zwar empirische Befunde für die Größe, Stabilität und beachtenswerte Leistungsfähigkeit der Wikis zusammen, doch fehlt bislang ein übergreifender Rahmen, welcher es erlaubt, jene in eine umfassende Erklärung des Phänomens zu integrieren. Die bisher veröffentlichten Arbeiten, die im Wesentlichen auf die umfangreiche Internetforschung in den Bereichen Computer Supported Cooperative Work (CSCW), Free/Libre/Open Source Software (FLOSS), netzbasierte Wissensproduktion, virtuelle Gemeinschaften und neuere Anwendungen onlinegestützter Kommunikation Bezug nehmen, gehen zumeist auf einzelne Aspekte der Kollaborationsprozesse ein. Im Fokus bisheriger Veröffentlichungen stehen insbesondere Strukturierungs- und Institutionalisierungsprozesse, Genreklassifikationen sowie die Informationsqualität der Artikel, Nutzertypologien, Motivlagen, informationstechnische Ansätze und Techniken zur Visualisierung der Interaktion. Dabei liegt der Schwerpunkt auf Untersuchungen der Wikipedia, welchen hier dementsprechend ein besonderer Raum in der Darstellung eingeräumt wird. Die Einordnungssystematik zur vorläufigen Gliederung der Ansätze folgt dabei keinem vorher definierten Prinzip, sondern wurde vielmehr in einem Bottom-up-Prozess im Verlauf der Sichtung der Beiträge gewonnen. Es handelt sich somit um eine jederzeit im Blick auf die Entwicklung des Feldes revidierbare Ad-hoc-Clusterung.

\subsection{Strukturierungstheoretische und genreklassifikatorische Ansätze}

Wikis, verstanden als netzbasierte kollaborative Systeme, sind ein willkommenes Feld für die Analyse technikvermittelter sozialer Prozesse und Strukturen (vgl. Kalz, 2005). Unter Berufung auf Giddens' Strukturierungstheorie (1984) wird Wikipedia als sich selbst organisierendes soziales Online-System verstanden, in dem die Autoren aktiv an der Strukturierung teilnehmen. Strukturierung bedeutet hierbei die Selbstorganisation von Regeln und Ressourcen und deren gleichzeitige Institutionalisierung. Es entsteht eine duale Struktur, in der das Handeln in gegebenen Strukturen auf diese wiederum zurückwirkt (vgl. grundlegend DeSanctis/Poole, 1994). Strukturen in Wikis sind somit Regel-Ressourcenkomplexe, welche von den Usern permanent weiterentwickelt werden. Dabei eröffnen sich weiterführende Untersuchungsdimensionen in Form von Herrschafts-, Signifikations- und Legitimationsprozessen.

In eine ähnliche Richtung gehen Versuche, das Wikipedia-Material, also die Artikeltexte, die umfangreichen Metatexte wie Diskussions- und Userseiten sowie die primär der Organisation der Community gewidmeten Teile, aus einer linguistischen Perspektive zu untersuchen (vgl. Emigh/Herring, 2005). Der Fokus liegt dabei auf dem Bestreben, das bereits erwähnte, von Giddens inspirierte Konzept, wonach jegliche Kommunikation in Genreformaten abläuft, anzuwenden (vgl. statt anderer Yates/Orlikowski, 1992). Die beiden entscheidenden, miteinander interagierenden Faktoren sind dabei die Nutzerwahlen und die Systemvoraussetzungen. Zur Analyse des Genres der Wikipedia als „enzyklopädischem Wissensspeicher“ (Emigh/Herring, 2005: 1) wird diese mit einem anderen kollektiv geschaffenen Online-Lexikon (everything2.com) und der digitalen Columbia Encyclopedia verglichen. Die Untersuchung konzentriert sich auf die Artikel 
und Diskussionsseiten und analysiert jene hinsichtlich des Formalisierungsgrades der Sprache, gemessen an der Häufigkeit von Kontraktionen ( $I^{\prime} m$, don't etc.), Personalpronomen und nomentypischen Suffixen (-ment, -(t)ion, -ity etc.). Den Hintergrund dafür bilden Arbeiten, welche darlegten, dass die ersten beiden Formen Indizien eines eher informellen Sprachgebrauches sind (vgl. z. B. Heylighen/Dewaele, 1999). Durchgeführt wurde die Studie an 15 Artikeln aus unterschiedlichen Gebieten (z. B. zu Karl Marx, Kandahar und Pizza) und deren Diskussionsseiten, welche in allen drei Enzyklopädien vorhanden waren. Neben den erwähnten Merkmalen wurde zudem die Länge des Textes und die durchschnittliche Länge der Wörter bestimmt. Dabei konnte gezeigt werden, dass Wikipedia zum Genre der Lexika zu zählen ist, da die Artikel einen hohen, mit gedruckten Enzyklopädien vergleichbaren Formalisierungsgrad der Sprache aufweisen, wohingegen die Diskussionen sehr informell sind und damit an Web-Boards und Foren erinnern. Durch ihre beständige Editierung erreichen die Artikel im Gegensatz zu everything2.com einen dem Genre des Lexikons entsprechenden Sprachstil. Grund dafür können möglicherweise die von den Wikipedia-Autoren internalisierten traditionellen Printnormen sein, die zudem im Editierprozess durch das Handeln der Community (z. B. durch Löschanträge für mangelhafte oder durch Auszeichnung exzellenter Artikel) verstärkt und in den Diskussionen gegebenenfalls expliziert werden.

\subsection{Handlungs- und konsensorientierte Ansätze}

Ein anderer Fokus richtet sich auf Wikis in ihrer Eigenschaft als asynchrones netzbasiertes Kommunikationssystem. In ihrer engen Verknüpfung von Artikeln und dazugehörigen Diskussionsseiten lösen sie sich von den starren Kommunikationsmustern der Foren, die jeweils nur eine beschränkte Themenwahl erlauben, durch die ThreadStruktur ${ }^{11}$ Bezugnahmen erschweren und keine Gelegenheit für Zusammenfassungen geben (vgl. Resnick et al., 2005).

Einige Forschungsansätze betonen die Produktivität der Wiki-internen Diskussionen im Prozess der Wissensgenerierung. Durch sie werde eine Form der soft security installiert, welche zum einen die Offenheit des Wiki-Systems gewährleiste und zum anderen die Daten wesentlich besser als restriktive Benutzerregeln schütze (vgl. Shah, 2005). ${ }^{12}$ Im Fall Wikipedia heißt dies: Die Speicherung jeder Editierversion erlaubt eine schnelle und einfache Revision böswilliger Änderungen oder Löschungen, während kontroverse Meinungen auf den Diskussionsseiten verhandelt werden können (vgl. auch Matei/Dobrescu, 2006; Lawler, 2005; 2005a; Pentzold, 2006).

Die Lösung von Konflikten nimmt in Wikipedia unterschiedliche Formen an: Zum einen können sich die Autoren auf einen Konsens einigen (in der Diskussion, mit Hilfe von Abstimmungen, durch Vermittlung Dritter), die divergierenden Positionen nebeneinander präsentieren oder das umstrittene Thema meiden. Zum anderen werden Auseinandersetzungen auch beendet durch eine Verdrängung und Löschung von Einträgen oder durch die (dauerhafte/temporäre) Sperrung von Nutzern, so dass sie nicht weiter am Editierprozess teilnehmen können (vgl. Pentzold/Seidenglanz, 2006).

Entscheidend für eine positive Funktion von Konflikten scheint deren integratives

11 Der Begriff thread stammt aus der Gesprächsanalyse und bezeichnet eine hierarchisch organisierte Folge von Diskussionsbeiträgen, bei der üblicherweise genau ein Thema diskutiert wird.

12 In einem größer gefassten Kontext bezeichnet das Konzept der soft security allgemein eine Form der Regulierung in sozialen Netzwerken, welche auf der gemeinschaftlichen Aushandlung geteilter Verhaltensstandards basiert. Vgl. Wikipedia:Soft_security. 
Potenzial innerhalb der Wikipedia zu sein (vgl. Reagle, 2004). Ausgangspunkt dieser Überlegung ist die Feststellung, dass Wissensgenerierung in Wikis immer Aushandlung bedeutet, Wikipedia also als kontinuierliche Folge von Kommunikationsakten beschrieben werden kann. Dass sie dennoch beständig wächst und neue Nutzer anzieht, statt im äußerst komplexen Geflecht der Änderungen und Diskussionen stehen zu bleiben, ist zumindest z. T. auf die erfolgreiche Lösung von Konflikten rückführbar. Eine befriedigende Erklärung dafür, warum die meisten Auseinandersetzungen in der Wikipedia zu einer Zusammenführung der Meinungen und nicht einem Zerstreuen der Ansichten führen, steht bislang aus (vgl. auch Reagle, 2005). ${ }^{13}$

Darüber hinaus bestehen Bestrebungen, die Offenheit des Produktions- und Kommunikationsprozesses innerhalb der Wikis als Einlösung der Forderung Enzensbergers nach einem emanzipatorischen Mediengebrauch (vgl. Ebersbach/Glaser, 2004) bzw. der Habermas'schen Idee des herrschaftsfreien Diskurses (vgl. Froomkin, 2003; Barton, 2005) zu werten.

Einen weiterführenden Blick auf die in Wikis ablaufenden Diskussionen eröffnet die Frage nach den Qualitätssicherungsmechanismen. Gerade Themen wie Richtigkeit und Gültigkeit der Informationen stehen derzeit im Zentrum der öffentlichen Auseinandersetzung um Wikipedia (vgl. die Kritik an der Arbeitsweise und den verfügbaren Informationen bei McHenry, 2004; Sanger, 2004 und polemisch Gorman, 2005). ${ }^{14}$

Die Informationsqualität von Wikipedia-Artikeln wurde bisher in einem ersten Schritt im Zusammenhang mit dem quantitativen Maß ,Bearbeitungsintensität ' untersucht, das über die Zahl der Editierversionen eines Artikels (rigor) und die Anzahl der daran beteiligten User (diversity) operationalisierbar scheint. Wenn beide Maße einen hohen Wert annehmen, also viele Nutzer einen Artikel oft redigieren, sei das ein Indikator für einen qualitativ hochwertigen Artikel. Zur Überprüfung dieser These wurden in einer Studie 333 Einträge analysiert, bevor und nachdem diese im Fernsehen oder in Zeitungen bzw. Zeitschriften zitiert wurden. Dabei konnte gezeigt werden, dass das Auftauchen in massenmedialen Kontexten zu einer gesteigerten Popularität einzelner Artikel führte. Dies wiederum äußerte sich neben erhöhten Zugriffszahlen auch im Ansteigen der Bearbeitungsintensität und damit im Wachstum des Beitrages (vgl. Lih, 2004). Unklar bleibt jedoch, inwieweit beide Maße Aufschluss über die Qualität der Informationen liefern. Auch scheint der problematische Begriff der Informationsqualität selbst nur ungenügend bestimmt zu sein.

An diesem Punkt versucht das Thema-Aufmerksamkeit-Qualität-Modell anzusetzen (vgl. Brändle, 2005). Die Artikelqualität wird dabei als abhängige Variable verstanden, die durch die Maße ,Themeneigenschaften` und ,Aufmerksamkeit‘ erklärt werden soll. Alle drei Dimensionen (Qualität, Thema, Aufmerksamkeit) setzen sich wiederum aus mehreren Variablen zusammen, welche sich mittels Faktorenanalyse in fünf Faktoren gliedern lassen: ,Relevanz des Themas', ,Beachtung', ,Kontroversität', ,Reichhaltigkeit und ,Neutralität‘. In multiplen Regressionsanalysen kann gezeigt werden, dass sich $64 \%$ $\left(\mathrm{R}^{2}=0.64\right)$ der Gesamtvarianz der Qualität eines Artikels durch die Maße ,Relevanz des Themas', ,Beachtung' und ,Reichhaltigkeit' aufklären lassen.

Eine andere Herangehensweise stellt die prozessorientierten Diskussionsseiten der

13 Bryant/Forte/Bruckman (2005) zeigen in ihrer Studie, wie sich Wikipedia-Novizen zu Experten entwickeln und dabei die Normen und Arbeitsweisen internalisieren.

14 Vgl. dazu auch journalistische Beiträge, wie etwa den Artikel „Unleserlicher Mist“ von Bernd Graff in der Süddeutschen Zeitung vom 7.12.2005. Ebenfalls kritisch Erik Malinowski in Wired „War of the Words“ (Ausgabe 13.12). 
Artikel in den Mittelpunkt (vgl. Stvilia et al., 2005; 2005a). Ein zentraler Punkt der Untersuchung ist die Feststellung, dass die Autoren in den Diskussionen zehn Informationsqualitätsprobleme benennen (Verständlichkeit, Richtigkeit, Einhaltung akademischer Standards, Vollständigkeit, Komplexität, Konsistenz, Informativität, Relevanz, Überprüfbarkeit und Unbeständigkeit) und jene auch in Prozessen der Qualitätssicherung (z. B. Aushandlung von strittigen Standpunkten, Löschantrag, Auszeichnung) als Argument oder Kritikpunkt mobilisieren. Vor dem Hintergrund dieser Feststellung wurden 60 zufällig gewählte Artikel und deren Diskussionsseiten betrachtet. Dabei zeigte sich, dass die Kategorie ,Richtigkeit' der wesentlichste Diskussionspunkt ist (57 Nennungen), gefolgt von ,Vollständigkeit‘ (49), ,Überprüfbarkeit‘ (19) und ,Relevanz (18). Damit sind sie mit gebräuchlichen Lexikon-Standards vergleichbar (vgl. auch Pentzold/Seidenglanz, im Erscheinen). Weiterhin liefert die Untersuchung eine der möglichen Bedingungen für die ausgesprochene Dynamik der Wikipedia: Das Vorhandensein einer kleinen, in ihren Meinungen relativ homogenen Kerngruppe von Autoren, die an einem Artikel mitarbeiten. Zwischen diesen kann sich im Prozess der kollaborativen Arbeit zum einen ein Gemeinschaftssinn, zum anderen eine verdienstbasierte soziale Ordnung entwickeln, in der Nutzern erweiterte Befugnisse aufgrund der Menge und Qualität ihrer Beiträge zugesprochen werden kann.

\subsection{Nutzerklassifikationen und Motivlagen}

Im Grunde soll die Herausbildung von Hierarchien in Wikis vermieden werden, da dies der Offenheit und Gleichberechtigung entgegenstünde. Gleichwohl entwickelt sich z. B. in der Wikipedia, wie bereits erwähnt, eine verdienstbasierte soziale Ordnung, in der sich entsprechend engagierte Nutzer um eine Reihe zusätzlicher Rechte bewerben können. Eine grundlegende Unterscheidung der Benutzer erfolgt in angemeldete und unangemeldete Autoren, Administratoren, Bürokraten, Stewards und Entwickler. Während die Autoren Artikel lesen und bearbeiten können, verfügen die aus ihnen mittels Abstimmung gewählten Administratoren bzw. Systemoperatoren (sysops) beispielsweise über die Befugnis zur vollständigen Artikellöschung inklusive des dazugehörigen Lemmas. Bürokraten und Stewards fungieren auf einer Ebene außerhalb der Artikelproduktion. So sind Bürokraten z. B. für die Zuweisung des Administratorstatus zuständig, wohingegen Stewards wiederum die Befugnisse des Bürokratenstatus freischalten können. Systemrelevante Aufgaben, wie etwa die Modifikation der MediaWiki-Software oder die Überprüfung der Wikipedia-Server, werden von den Entwicklern wahrgenommen. Zur endgültigen Schlichtung von Konflikten ist schließlich der Vermittlungsausschuss gefragt, der mithin die letzte Entscheidungsinstanz darstellt (vgl. Wikipedia:Benutzer).

Darüber hinaus benennt eine erweiterte Nutzerklassifikation des Wikipediagründers Wales (2004) ferner: sozial orientierte Typen (socialites and trolls), Artikelschreiber (worker bees and point-of-view-pushers), Kontrolleure (police and judges), Streitsuchende (moths), Pseudo-User (sock puppets and vandals) und User in einer Kategorie Extrawiki, die sich außerhalb der eigentlichen Artikelproduktion befindet (mailing lists, IRC, board activities, developers). Zusammen bilden sie eine ,Gemeinschaft bedachter Benutzer' - eine Vorstellung, die der Idee von Wikipedia als emergentem Phänomen ohne erkenn- und benennbare Voraussetzungen und Gründe widerspricht. Untermauert wird die These durch die sog. 80/10-Regel: $10 \%$ aller User produzieren $80 \%$ aller Einträge. D. h. obwohl die Wikipedia eine enorme Zahl angemeldeter Autoren aufweisen kann, trägt eine im Vergleich dazu relativ kleine Gruppe maßgeblich zum Wachstum und zur Stabilität der Enzyklopädie bei. So zeigt ein Blick in die Autorenstatistik, dass zwar 
derzeit mehr als zwei Millionen Nutzer in der englischen Version registriert sind, doch im Juni 2006 nur ca. 111.000 von ihnen aktiv mit mehr als zehn Beiträgen teilgenommen haben (vgl. Zachte, 2006 und aus systemwissenschaftlicher Perspektive Frost, 2006). ${ }^{15}$

Neben der Idee einer community of thoughtful users können Wikis allgemein in Anlehnung an Wenger (1999) und Nonaka/Takeuchi (1995) als communities of practice verstanden werden, in denen das gemeinsame Engagement der Benutzer Zusammenhalt schafft. Interaktivität ist demnach eine Vorraussetzung für die Entwicklung von Gemeinschaftssinn als möglichem Motiv der Wiki-Benutzer. Unter dieser Perspektive versucht ein transkulturelles Online-Survey erste Ansätze zur Beschreibung der Nutzer-Motivation in Wikipedia zu liefern (vgl. Rafaeli/Hayat/Ariel, 2005). Insgesamt 120 Wikipedianer (85 englischsprachige, 35 hebräischsprachige) wurden befragt und dabei die Motivation anhand von acht Items gemessen: ,Zusammenarbeit mit anderen', das ,Lernen neuer Dinge‘, ,Diskussion', ,Zeit verbringen', die ,intellektuelle Herausforderung', ,Freude', das ,Teilen von Wissen' und die ,Reputation auf einem speziellen Gebiet'. Die Ergebnisse zeigen, dass die wichtigsten kognitiven Motive das ,Lernen neuer Dinge' und die ,intellektuelle Herausforderung' sind. Eine wichtige Rolle spielen auch integrative (,Teilen von Wissen', ,Zusammenarbeit mit anderen') und affektive Motive (,Freude).

Zwei andere Erhebungen, die sich auf Daten einer internationalen Onlinebefragung unter 525 Wikipedia-Autoren (davon 106 aus Deutschland) stützen, betonen die Identifikation mit dem Projekt und Tätigkeitscharakteristika der kollaborativen Wissensgenerierung (vgl. Schroer/Hertel, 2006; Schroer et al., 2005). Erhoben wurden sowohl demografische Daten und motivationale Prozessvariablen (intrinsisch, extrinsisch, Identifikation) als auch Form, Dauer, Inhalt und die Konsequenzen des Engagements für Wikipedia. Als Ergebnis lassen sich vor allem intrinsische Motivationsfaktoren (Lernen, Spaß, Flow-Erlebnis) benennen. Auch die Optimierung der Artikelqualität und das Schaffen eines bleibenden Guts stellen motivationale Faktoren dar. Schließlich spielen die Identifizierung mit dem Projekt und der Wunsch, Wikipedia kontinuierlich zu verbessern, eine wichtige Rolle. ${ }^{16}$

Ein Rückgriff auf die ökonomische Klub-Theorie (vgl. Scotchmer, 2002) ist hingegen wenig Erfolg versprechend, wenn es um die Erklärung der Motivlagen geht. Entgegen deren theoretischer Annahme, dass die Produktivität sinkt, wenn die Produzentenzahl ein Optimum überschreitet, lässt das stetige Wachstum der Wikipedia bei gleichzeitigem Ansteigen der Autorenzahlen vermuten, dass ein beständiges Gruppenwachstum nicht

15 Gelegentlich wird das Verhältnis auch als 90/10- oder 80/20-Regel bezeichnet - je nachdem, welcher Datensatz zugrunde liegt und wie das Kriterium ,aktiver Nutzer' gedeutet wird. Zudem erstellen die Wikipedia-Autoren selbst umfangreiche Nutzer-Klassifikationen, in denen sie sich selbst verschiedenen Kategorien zuordnen, wobei diese keinen wissenschaftlichen Systematisierungsansprüchen folgen und die Etikettierungen gelegentlich einen eher scherzhaften Eindruck machen (vgl. Wikipedia:Wikipedians und Wikimedia:The Wikipedia Community). Da Lio/Fraboni/Leo (2005) legen eine Klassifikation von Nutzerrollen in einem anderen, von Wikipedia verschiedenen Wiki vor.

16 Vgl. auch zu Motivlagen in Open-source-Projekten Hertel/Niedner/Herrmann (2003), die in ihrer Befragung von Entwicklern des Linux kernel (elementarer Kern des Linux-Betriebssystems) ebenfalls die Wichtigkeit von intrinsischen, auf den Gemeinschaftsaspekt gerichteten Motiven zeigen können. Neben den Fragebogenuntersuchungen wird auch ein möglicher Ansatz entwickelt, etwaige Motive durch gesteuerte Eingriffe in die Wikipedia-Kollaborationsprozesse aufzuzeigen. Bislang scheint die Studie jedoch noch nicht über das Entwurfsstadium hinausgekommen zu sein (vgl. Tobacman, 2005). 
in allen Fällen kontraproduktiv sein muss (vgl. Ciffolilli, 2003). Stattdessen verweisen die niedrigen Transaktionskosten der Teilnahme, das Fehlen des Faktors ,Eigennutz und das Ausbleiben einer Bezahlung auf intrinsische Motive wie Wissenserwerb und Lob. Des Weiteren kann als soziales Motiv zur Partizipation der Wunsch genannt werden, zu einer Gruppe zu gehören und an der Produktion eines gemeinsamen Guts teilzunehmen. Extrinsische Motive zielen auf mögliche Referenzen für den Arbeitsmarkt und den Aufstieg innerhalb der Community ab.

Eine Schwierigkeit für die eben skizzierten Erklärungen der Motivlagen stellt die z. T. hohe Anzahl an anonymen Autoren dar, die nur durch eine von ihrem Internet-Provider zugewiesene IP-Adresse unterscheidbar sind. Eine Vielzahl dieser Benutzer leistet einen erheblichen Beitrag zur Konstruktion der Artikel. ${ }^{17}$ Auch lässt sich bisher kein empirischer Beleg für einen Zusammenhang zwischen Editiermustern und Graden der Identitätspreisgabe ausmachen, d. h., Vandalismus wird z. B. nicht nur von anonymen Nutzern betrieben (vgl. Ma, 2005; Anthony/Smith/Williamson, 2005).

\subsection{Visualisierungstechniken und weitere informationstechnische Ansätze}

Ein weiteres wesentliches Forschungsfeld stellt die Aufzeichnung der kollektiven Arbeit in Wikis dar. Die History-flow-Visualisierung bildet dabei den bisher wichtigsten Beitrag (vgl. Viégas/Wattenberg/Dave, 2004). Diese am MIT Media Lab und IBM Research Lab programmierte Software ermöglicht es, die dynamische Entstehung und damit die Kollaborationsmuster von Wikipedia-Artikeln grafisch abzubilden (vgl. auch ähnliche Ansätze wie Theme River ${ }^{T M}$ von Havre/Hetzler/Nowell, 1999 und die Clear-Eye-Software von Kong/Zheng, 2005). Dargestellt werden die Userbeiträge mit unterschiedlichem Textumfang (farbig), deren Datum und gegebenenfalls der Kommentar, mit dem die Änderung in der Artikelhistorie versehen ist (Abb. 2).

Problematisch ist hierbei, dass die unterste Untersuchungseinheit Sätze sind, kleine Änderungen also als ganzer Satz angezeigt werden. Als eines der ersten Ergebnisse ihrer Untersuchung liefern Viégas, Wattenberg und Dave eine Klassifikation von Störungen der Artikelproduktion. Dabei unterscheiden sie mass deletion ( $90 \%$ des Artikels gelöscht, kurze Bestehenszeit dieser Löschung, das Wort „fuck“ im verbliebenen Textfragment enthalten), offensive copy (vulgäres, beleidigendes o. ä. Material in Artikel eingearbeitet), phony copy (themenfremdes, nicht zum Lemma passendes Material eingefügt), phony redirect (Umleitung zu themenfremder, nicht mit dem Lemma verbundener Seite) und idiosyncratic copy (Einschub tendenziöser, wenngleich nicht unbedingt themenfremder Beiträge). Darüber hinaus können sie zeigen, dass während so genannter edit wars, d. h. der länger andauernden Löschung bzw. Wiederherstellung eines Artikelteiles, die Diskussionen auf den entsprechenden Seiten eskalieren können. Als Desiderate nennen sie die Formulierung eines besseren Such-Algorithmus für die Erkennung von Vandalismus, die Durchführung von kulturvergleichenden Studien sowie eine systematische Untersuchung der dualen Struktur von Artikel und dazugehöriger Diskussionsseite.

Weitere ebenfalls informationstechnische Ansätze befassen sich mit der Hyperlinkstruktur von Beiträgen (vgl. Bellomi/Bonato, 2005; Grangier/Bengio, 2005; Capocci et

17 Im Vergleich wird ersichtlich, dass die Anzahl der anonymen, jedoch aktiv mitwirkenden User in verschiedenen Sprachausgaben unterschiedlich stark ausgeprägt ist. So werden in der japanischen Wikipedia ca. $42 \%$, in der deutschen $26 \%$, in der englischen $22 \%$ und in der französischen bspw. nur $15 \%$ aller Editierungen von anonymen Usern vorgenommen (vgl. Voß, 2005: 9). 
Abbildung 2: Eine von der History-flow-Visualisierung erstellte Darstellung der prototypischen Bearbeitungsvorgänge in Wikis

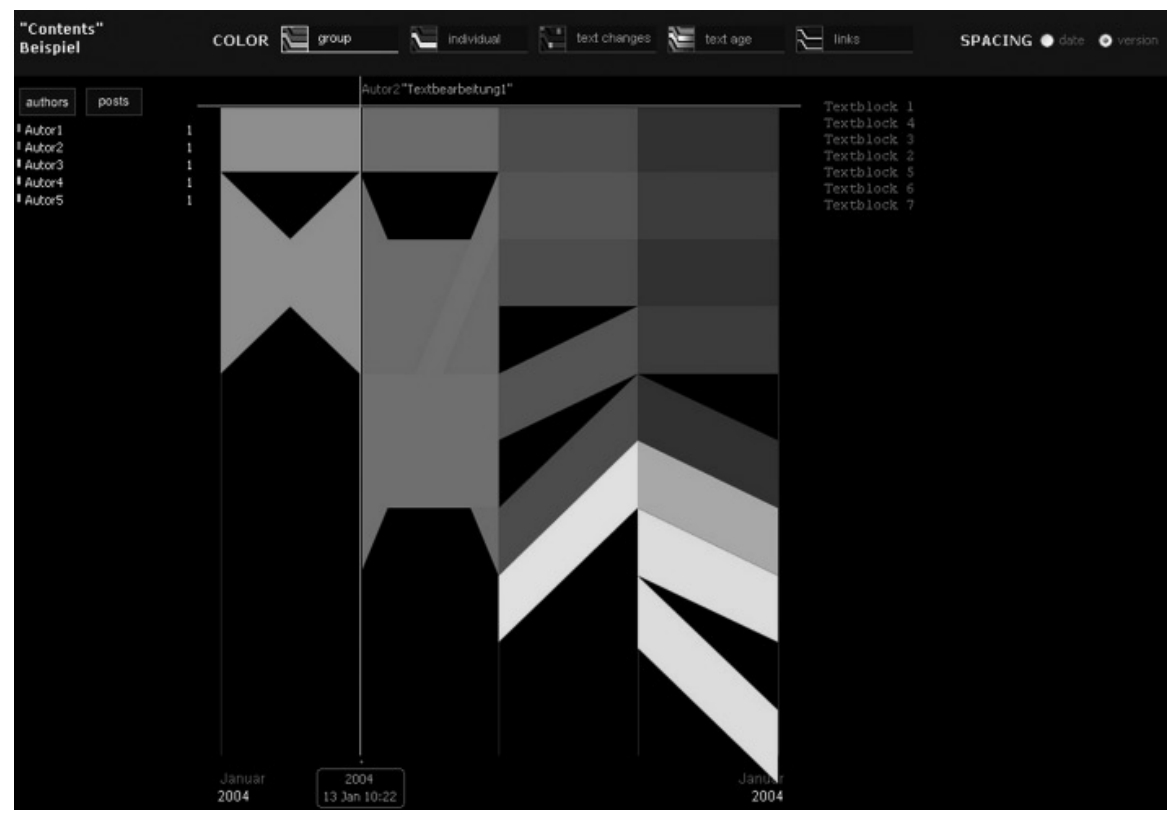

Auf einem Zeitstrahl werden die Nutzer mit ihren größenmäßig verschiedenen Beiträgen dargestellt. Deutlich zu sehen ist die Verschiebung und Hinzufügung neuer Textblöcke durch zunächst fünf User.

Quelle: Sebastian Seidenglanz, Visualisierung eines Test-Wikis, Professur Mediennutzung, TU Chemnitz, 14.08.2006.

al., 2006; Zlatić et al., 2006), der generellen Entwicklung der Wiki-Software (vgl. u. a. Di Iorio/Vitali, 2005; Désilets et al., 2006) und der Verbindung von Wikis mit dem Semantic Web (vgl. zur grundlegenden Idee Berners-Lee/Hendler/Lassila, 2001 und im Speziellen Krötzsch/Vrandečić/Völkel, 2005; Harth et al., 2005). In eine andere Richtung gehen Versuche, den Inhalt von Wikis nach semantischen Kriterien in Karten darzustellen und somit eine Art themengebundener Topografie zu entwickeln (vgl. Holloway/Božičević/ Börner, 2005).

\section{Wissensgenerierung in Wikis - Skizzierung eines Analyserahmens für Wikipedia}

Der Überblick zum Stand der Forschung zeigt, dass eine Reihe von Ansätzen existiert, Eigenschaften und Arbeitsweisen von Wikis und im Speziellen von Wikipedia zu beschreiben. Jedoch werden auch die Forschungslücken deutlich: Keiner der skizzierten Beiträge kommt über eine deskriptive Beschreibung oder eine partielle Erklärung des Phänomens hinaus. Von theoretischer Seite mangelt es an einer umfassenden Perspektive, die zum einen der Wikipedia-Struktur als Kombination aus Artikeln, Diskussionsseiten und Metadokumenten gerecht wird, und zum anderen der Interaktion der Nutzer in Wissensgenerierungs- und Aushandlungsprozessen hinreichend Rechnung 
trägt. Die Strukturierungstheorie kann in ihren empirischen Analysen der Kommunikationsgenres bislang kaum die dynamischen Prozesse der Wissensgenerierung erläutern, während die an den Diskussionen und Aushandlungsprozessen orientierten Ansätze vornehmlich deskriptiv vorgehen. Auch die Studien zur Informationsqualität, zu Motivlagen und Nutzertypologien beschränken sich auf Ausschnitte des Forschungsfeldes. In empirischer Hinsicht stellen die bisher konstruierten Visualisierungstechniken ein interessantes, ausbaufähiges Werkzeug dar, ohne jedoch Erklärungen für die beobachteten Dynamiken anbieten zu können.

In Abgrenzung dazu kann ein Analyserahmen aufgespannt werden, welcher mit einer Integration system- und diskurstheoretischer Methoden die bisherigen, vorwiegend deskriptiven Ansätze um eine theoriegeleitete, selektiv-beobachtende Perspektive mit explikativem Anspruch erweitert. Dabei wird von der Annahme ausgegangen, dass sich die Entstehung und Veränderung von Wiki-Texten als Folge des Operierens sozialer Kommunikationssysteme erklären lässt (vgl. Luhmann, 1984; 1997). Versteht man Wikipedia in diesem Sinne als paradigmatisches Forschungsfeld zur Untersuchung sozialer Systeme, dann eröffnet sich die Gelegenheit, die Zusammenhänge und Dynamiken zwischen 1. sich stabilisierenden und reproduzierenden Prozessen in emergenten Kommunikationssystemen, 2. sozialen Netzwerken und 3. der Produktion kulturellen Wissens zu untersuchen. Auf diese Weise wird nicht nur ein leistungsfähiger Ansatz zur Erklärung der Funktionsweisen der social software Wikipedia formuliert, sondern es wird darüber hinausgehend auch eine fruchtbringende Verbindung zwischen Diskurs- und Systemtheorie auf der Mikro- und Makrountersuchungsebene hergestellt.

Die Ziele eines solchen Vorgehens werden konzeptuell von zwei Seiten her in Angriff genommen, die sich gegenseitig ergänzen und bedingen: Zum einen soll die Produktion von Wikipedia-Artikeln soziologisch-systemtheoretisch (vgl. Willke, 1993) erklärt werden. Zum anderen sollen die Prozesse der Selbststabilisierung von autopoietischen Kommunikationssystemen anhand der Kommunikation in Wikipedia analysiert und die Ergebnisse in einem generalisierbaren, dynamisierbaren Modell formuliert werden. Ausgangspunkt dieser Überlegungen ist die duale Struktur der Artikel, bestehend aus dem eigentlichen enzyklopädischen Text und den Diskussionsseiten, welche in ihrer Vollständigkeit von der MediaWiki-Software dokumentiert sind und somit abrufbar bereitliegen. In Anlehnung an Luhmann ist davon auszugehen, dass sich auch in dieser Struktur exemplarisch Systemkommunikation nachweisen lässt, die im Hinblick auf ihre Funktion als universell erforderliche Grundoperation in allen sozialen Systemen vorkommen muss. Das beabsichtigte Ziel soll es sein, am Beispiel der Wikipedia-Kommunikation die systemtheoretisch postulierten Merkmale selbstreferentieller Kommunikation $\mathrm{zu}$ analysieren und ihre systemkonstituierende Funktion zu spezifizieren. Vor diesem Hintergrund wird die Erstellung eines Artikels als komplexitätsreduzierender Systemdifferenzierungsprozess verstanden. Dieser ist in seinen charakteristischen Phasen in zwei Dimensionen analysierbar: inhaltlich (in den Texten) und auf der Netzwerk-Ebene (zwischen Autoren). Dazu werden zum einen mittels inhaltsanalytischer Verfahren (vgl. u. a. Strauss/Corbin, 1996; Merten, 1995) in den Artikeln und Diskussionen Textmerkmale, die auf thematische Strukturierung hinweisen, herausgearbeitet. Daneben dient eine Autoren-Netzwerkanalyse dazu, mittels graphentheoretischer Parameter die Strukturdifferenzierungsprozesse im Gesamtnetzwerk zu bestimmen (vgl. Seidenglanz/Pentzold, 2006). Die Ergebnisse dieser Analysen wiederum sollen in einem nächsten, noch zu leistenden Schritt in eine integrative Untersuchung des Aufbaus kollektiv generierter und geteilter Wissensbestände, der System-Kommunikation, die zum Aufbau dieser Wissensbestände geführt hat, und der Struktur des sozialen Netzwerkes, 
in dem sich diese Kommunikation entfaltet, in ihrer Interdependenz und Entwicklungsdynamik münden.

\section{Literaturverzeichnis}

Alexa.com [Online] alexa.com (14.08.2006).

Andersen, E. (2004): Using Wikis in a Corporate Context. [Online] www.espen.com/papers/Andersen-2005-corpwikis.pdf (14.08.2006).

Anthony, D./S. W. Smith/T. Williamson (2005): Explaining Quality in Internet Collective Goods. Zealots and Good Samaritans in the Case of Wikipedia. [Online] http://web.mit.edu/iandeseminar/Papers/Fall2005/anthony.pdf (14.08.2006).

Aronsson, L. (2002): Operation of a Large Scale, General Purpose Wiki Website. In: J. A.Carvalho/A. Hübler/A. A. Baptista (Hg.): Elpub 2002. Technology Interactions. Berlin: Verlag für Wissenschaft und Forschung, 27-37.

Augar, N./R. Raitman/W. Zhou (2004): Teaching and Learning Online with Wikis. In: R. Atkinson et al. (Hg.): Beyond the Comfort Zone. Proceedings of the $21^{\text {st }}$ ASCILITE Conference, Perth, 95-104. [Online] www.ascilite.org.au/conferences/perth04/procs/augar.html (14.08.2006).

Barton, M. D. (2005): The Future of Rational-Critical Debate in Online Public Spheres. In: Computers and Composition, 22(2005), 177-190.

Bellomi, F./R. Bonato (2005): Network Analysis for Wikipedia. [Online] www.fran.it/articles/ wikimania_bellomi_bonato.pdf (14.08.2006).

Berners-Lee, T./J. Hendler/O. Lassila (2001): The Semantic Web. In: Scientific American, 234(5), $34-43$.

Boyd, S. (2003): Are you ready for Social Software? In: Darwin Mag, 5/03. [Online] www.darwinmag.com/read/050103.html (14.08.2006).

Brändle, A. (2005): Too Many Cooks Don't Spoil the Broth. In: Proceedings of the Wikimania'05, Frankfurt a. M. Wikimania-Paper AB1. [Online] http://en.wikibooks.org/w/index. php?title=Wikimania05/AB1 (14.08.2006).

Bruns, A./S. Humphreys (2005): Wikis in Teaching and Assessement: The M/Cyclopedia Project. In: Proceedings of the WikiSym'05, San Diego.

Bryant, S. L./A. Forte/A. Bruckman (2005): Becoming Wikipedian: Transformation of Participation in a Collaborative Online Encyclopedia. Vortrag auf der GROUP'05, Sanibel Island, Florida.

Capocci, A. et al. (2006): Preferential Attachment in the Growth of Social Networks: The Case of Wikipedia. [Online] http://arxiv.org/PS_cache/physics/pdf/0602/0602026.pdf (15.01.2007).

Chawner, B./P. H. Lewis (2004): WikiWikiWebs: New Ways of Interacting in a Web Environment. In: Proceedings of the LITA National Forum, St. Louis. [Online] www.ala.org/ala/lita/ litaevents/2004Forum/CS_WikiWikiWebs.pdf (14.08.2006).

Ciffolilli, A. (2003): Phantom Authority, Self-Selective Recruitment and Retention of Members in Virtual Communities. The Case of Wikipedia. In: First Monday, 8(12). [Online] http://firstmonday.org/issues/issue8_12/ciffolli/index.html (14.08.2006).

Da Lio, E./L. Fraboni/T. Leo (2005): TWiki-based Facilitation in a Newly Formed Academic Community of Practice. In: Proceedings of the WikiSym'05, San Diego.

Danowski, P./J. Voß (2005): Das Wissen der Welt - Die Wikipedia. In: B. Lutterbeck/ R. A. Gehring/M. Bärwolff (Hg.): Open Source Jabrbuch 2005. Zwischen Softwareentwicklung und Gesellschaftsmodell. [Online] http://eprints.rclis.org/archive/00003351/01/osjb2005-06-05danowskivoss.pdf (14.08.2006).

Davies, W. (2003): You don't know me, but... Social Capital \& Social Software. Research paper der iSociety. [Online] http://theworkfoundation.co.uk/pdf/1843730103.pdf (14.08.2006).

DeSanctis, G./M. S. Poole (1994): Capturing the Complexity in Advanced Technology Use: Adaptive Structuration Theory. In: Organization Science, 5(2), 121-147.

Désilets, A./S. Paquet/N. G. Vinson (2005): Are Wikis Usable? In: Proceedings of the WikiSym'05, San Diego. 
Désilets, A. et al. (2006): Translating the Wiki Way. In: Proceedings of Wikisym'06. San Diego: ACM Press, 19-31.

Di Iorio, A./F. Vitali (2005): From the Writable Web to Global Editability. In: Proceedings of the $H T^{\prime} 0$ S, Salzburg. [Online] http://portal.acm.org/citation.cfm?id=1083365\&dl=acm\&coll=\&C $\mathrm{FID}=15151515 \& \mathrm{CFTOKEN}=6184618$ (14.08.2006).

Döbeli Honegger, B. (2005): Wikis - A Rapidly Growing Phenomenon in the German-Speaking School Community. In: Proceedings of WikiSym'05, San Diego.

Ebersbach, A./M. Glaser (2004): Towards Emancipatory Use of a Medium. The Wiki. In: International Journal of Information Ethics, 2(11). [Online] http://container.zkm.de/ijie/ijie/ no002/ijie_002_09_ebersbach.pdf (14.08.2006).

Ebersbach, A./M. Glaser/R. Heigl (2005): WikiTools. Kooperation im Web. Berlin, Heidelberg, New York: Springer.

Emigh, W./S. C. Herring (2005): Collaborative Authoring on the Web: A Genre Analysis of Online Encyclopedias. In: Proceedings of the $38^{\text {th }}$ Hawaii International Conference on System Sciences (HICSS'05), Waikoloa, Hawaii. [Online] http://ieeexplore.ieee.org/ iel5/9518/30166/01385436.pdf?arnumber=1385436 (14.08.2006).

FDL (2002): GNU Free Documentation License. [Online] www.gnu.org/licenses/fdl.html\#SEC1 (14.08.2006).

Fiebig, H. (2005): Wikipedia. Das Buch. Berlin: Zenodot.

Flickr.com [Online] flickr.com (12.01.2007).

Forte, A./A. Bruckman (2006): From Wikipedia to the Classroom: Exploring Online Publication and Learning. In: Proceedings of the $7^{\text {th }}$ International Conference on Learning Sciences, Bloomington/IN. [Online] http://portal.acm.org/ft_gateway.cfm?id=1150061\&type=pdf\&col $l=$ GUIDE\&dl=GUIDE\&CFID=15151515\&CFTOKEN=6184618 (14.08.2006).

Fraas, C. (2005): Diskurse on- und offline. In: Dies./M. Klemm (Hg.): Mediendiskurse. Bestandsaufnabme und Perspektiven. Frankfurt a. M.: Peter Lang, 83-103.

Froomkin, A. M. (2003): Habermas@Discourse.net: Toward a Critical Theory of Cyberspace. In: Harvard Law Review, 116(3), 751-871.

Frost, I. (2006): Zivilgesellschaftliches Engagement in virtuellen Gemeinschaften. Eine systemwissenschaftliche Analyse des deutschsprachigen Wikipedia-Projektes. München: Herbert Utz.

Frumkin, J. (2005): The Wiki and the Digital Library. In: OCLC Systems E Services, 21(1), 18-22.

Fuchs-Kittowski, F./A. Köhler (2005): Wiki Communities in the Context of Work Processes. In: Proceedings of the WikiSym'05, San Diego.

Garcia, J. M./W. E. Steinmueller (2003): Applying the Open Source Development Model to Knowledge Work. INK Working Paper 2. [Online] http://siepr.stanford.edu/programs/ OpenSoftware_David/oswp2.pdf (25.01.2007).

Giddens, A. (1984): The Constitution of Society. Outline of the Theory of Structuration. Cambridge: Polity Press.

Giles, J. (2005): Internet Encyclopedias Go Head to Head. In: Nature, Dec. 05. [Online] www. nature.com/nature/journal/v438/n7070/full/438900a.html (14.08.2006).

Gorman, G. E. (2005): Is the Wiki Concept Really so Wonderful? In: Online Information Review, 29(3), 225-226.

GPL (1991): GNU General Public License. [Online] http://www.gnu.de/gpl-ger.pdf (14.08.2006).

Graff, B. (2005): Unleserlicher Mist. In: Süddeutsche Zeitung, 07.12.2005. [Online] http://www. sueddeutsche.de/kultur/artikel/772/65707/print.html (14.08.2006).

Grangier, D./S. Bengio (2005): Inferring Document Similarity from Hyperlinks. In: Proceedings of the CIKM'O5, Bremen.

Groß, M./W. Hülsbusch (2004): Weblogs und Wikis - eine neue Medienrevolution? In: Wissensmanagement, 8/04, 44-48.

Harth, A. et al. (2005): WikiOnt: An Ontology for Describing and Exchanging Wikipedia Articles. In: Proceedings of the Wikimania'05, Frankfurt a. M. Wikimania-Paper IM1. [Online] http://en.wikibooks.org/w/index.php?title=Wikimania05/IM1 (14.08.2006).

Havre, S./B. Hetzler/L. Nowell (1999): ThemeRiver ${ }^{T M}$ : In Search of Trends, Patterns, and Relationships. [Online] http://citeseer.ist.psu.edu/havre99themerivertm.html (14.08.2006). 
Hepp, A. (2006): Wissenspraktiken im Alltag: Wikipedia und Podcasting zwischen Konnektivität und Mobilität. In: K. Pühringer/S. Zielmann (Hg.): Vom Wissen und Nicht-Wissen einer Wissenschaft. Kommunikationswissenschaftliche Domänen, Darstellungen und Defizite. Münster: LIT, 171-194.

Hertel, G./S. Niedner/S. Herrmann (2003): Motivation of software developers in Open Source projects. An internet-based survey of contributors to the Linux kernel. In: Research Policy, 32, 1159-1177.

Heylighen, F./J.-M. Dewaele (1999): Formality of Language: Definition, Measurement and Behavioral Determinants. Interner Bericht, Center "Leo Apostel", Vrije Universiteit Brüssel.

Holloway, T./M. Božičević/K. Börner (2005): Analyzing and Visualizing the Semantic Coverage of Wikipedia and its Authors. Submitted to Complexity. [Online] http://arxiv.org/ftp/cs/papers/0512/0512085.pdf (14.08.2006).

Kalz, M. (2005): Strukturierungstheorie: ein Rahmenwerk für die Wikiforschung. In: Proceedings of the Wikimania'05, Frankfurt a. M. Wikimania-Paper MK3. [Online] http://en.wikibooks. org/wiki/Wikimania05/Paper-MK3 (14.08.2006).

Kohl, C./W.-A. Liebert (2004): Selbstorganisation in der Wissensvermittlung. Quellentransparenz, Kontroversität und Qualitätssicherung in der Internet-Enzyklopädie Wikipedia. In: Fachsprache, 26(3/4), 133-148.

Kong, Q./Q. Zheng (2005): Clear Eye. A Visualization System for Document Revision. CPSC 533C Project. [Online] www.cs.ubc.ca/ qkong/css/cpsc533c_report.htm (14.08.2006).

Krötzsch, M./D. Vrandečić/M. Völkel (2005): Wikipedia and the Semantic Web - The Missing Links. In: Proceedings of the Wikimania'05, Frankfurt a. M. Wikimania-paper MK2. [Online] http://en.wikibooks.org/wiki/Wikimania05/Paper-MK2 (14.08.2006).

Lange, C. (Hg.) (2005): Wiki. Planen, Einrichten, Verwalten. Böblingen: C.\&L.

Lawler, C. (2005): Wikipedia as a Learning Community. Unveröff. Master Thesis, University of Manchester.

Lawler, C. (2005a): Wikipedia as a Learning Community: Content, Conflict and the ,Common Good'. In: Proceedings of the Wikimania'05, Frankfurt a. M. Wikimania-Paper CL1. [Online] http://en.wikibooks.org/wiki/Wikimania05/Paper-CL1 (14.08.2006).

Leitner, H. (2003): Online-Community, „Hands On“! Ein Streifzug durch die Welt der OnlineCommunities. In: C. Eigner et al.: Online-Communities, Weblogs und die soziale Rückeroberung des Netzes. Graz: Nausner \& Nausner, 11-51.

Leuf, B./W. Cunningham (2001): The Wiki Way. Quick Collaboration on the Web. Boston et al.: Addison-Wesley.

Lievrouw, L. A. (2002): Determination and Contingency in New Media Development: Diffusion of Innovations and Social Shaping of Technology Perspectives. In: Dies./S. Livingstone (Hg.): Handbook of New Media. Social Shaping and Consequences of ICTs. London, Thousand Oaks, Neu Delhi: Sage, 183-199.

Lih, A. (2004): Wikipedia as Participatory Journalism: Reliable Sources, Matrix for Evaluating Collaborative Media as a News Resource. In: Proceedings of the $5^{\text {th }}$ International Symposium on Online Journalism, Austin. [Online] http://journalism.utexas.edu/onlinejournalism/2004/ papers/wikipedia.pdf (14.08.2006).

Luhmann, N. (1984): Soziale Systeme. Grundriß einer allgemeinen Theorie. Frankfurt a. M.: Suhrkamp.

Luhmann, N. (1997): Die Gesellschaft der Gesellschaft. Frankfurt a. M.: Suhrkamp.

Lund, A./O. Smødal (2006): Is There a Space for the Teacher in a WIKI? In: Proceedings of the WikiSym'06, San Diego: ACM Press, 37-46.

Ma, C. (2005): Wikipedia - Anonymous Users as Good Users. In: Proceedings of the Wikimania'05, Frankfurt a. M. Wikimania-Paper CM1. [Online] http://en.wikibooks.org/wiki/Wikimania05/Paper-CM1 (14.08.2006).

Malinowski, E. (2005): War of the Words. The Topics Wikipedians Never get Tired of Fighting Over. In: Wired Magazine, 13(12). [Online] http://www.wired.com/wired/archive/13.12/start. html?pg=14 (14.08.2006). 
Matei, S. A./C. Dobrescu (2006): Ambiguity and Conflict in the Wikipedian Knowledge Production System. Paper presented at the ICA Annual Meeting '06, Dresden. [Online] http://www. matei.org/ithink/papers/ambiguity-conflict-wikipedia/ (14.08.2006).

McHenry, R. (2004): The Faith-Based Encyclopedia. [Online] http://www.tcsdaily.com/article. aspx?id=111504A (14.08.2006).

Merten, K. (1995): Inhaltsanalyse. Einführung in Theorie, Methode und Praxis. ${ }^{2}$ Opladen: Westdeutscher Verlag.

Miller, N. (2005): Wikipedia and the Disappearing “Author”. In: ETC: A Review of General Semantics, 62(1), 37-40.

Möller, E. (2005): Die heimliche Medienrevolution. Wie Weblogs, Wikis und freie Software die Welt verändern. Hannover: Heise.

MySpace.com [Online] myspace.com (12.01.2007).

Nonaka, I./H. Takeuchi (1995): The Knowledge-Creating Company. Oxford: Oxford University Press.

Nupedia (2000): How to be an Editor or Peer Reviewer for Nupedia. [Online] http:// nupedia.8media.org/steering.shtml (14.08.2006).

Paquet, S. (2003): A Social-Technological Approach to Sharing Knowledge across Disciplines. Unveröff. Ph.D. Thesis. Université de Montréal.

Pentzold, C. (2006): Dispositiv, Diskurs, Diskursfragment. Zum Vorschlag eines diskurstheoretischen Analyserahmens für die Online-Enzyklopädie Wikipedia. In: LINSE - LinguistikServer Essen. [Online] http://www.linse.uni-essen.de/linse/publikationen/foucault_wiki.html (03.01.2007).

Pentzold, C./S. Seidenglanz (2006): Foucault@Wiki. First Steps Towards a Conceptual Framework for the Analysis of Wiki Discourses. In: Proceedings of the Wikisym'06 (Odense, Dänemark, 21.-23.08.2006). San Diego: ACM Press, 59-68.

Pentzold, C./S. Seidenglanz (im Erscheinen): Die aktuelle Enzyklopädie - Wikipedia im Spannungsfeld von Lexikaproduktion und Nachrichtenereignissen. In: Proceedings der 2. Studentischen Medientage Chemnitz, 31.03.-01.04.2006.

Portland Pattern Repository. http://c2.com/cgi/wiki (14.08.2006).

Rafaeli, S./T. Hayat/Y. Ariel (2005): Wikipedians' Sense of Community, Motivations, and Knowledge Building: A Cross-Cultural Study. In: Proceedings of the Wikimania'05, Frankfurt a. M. Wikimania-Paper YA1. [Online] http://en.wikibooks.org/wiki/Wikimania05/Paper-YA1 (14.08.2006).

Raymond, E. S. (2000): The Cathedral and the Bazaar. Version 3.0. [Online] http://www.catb. org/\%7Eesr/writings/cathedral-bazaar/ (14.08.2006).

Reagle, J. M. (2004): A Case of Mutual Aid: Wikipedia, Politeness, and Perspective Taking. [Online] http://reagle.org/joseph/2004/agree/wikip-agree.html (14.08.2006).

Reagle, J. M. (2005): Ph.D. Research Proposal. [Online] http://reagle.org/joseph/2005/disp/1proposal.html (14.08.2006).

Resnick, P. et al. (2005): Beyond Threaded Conversation. In: Proceedings of the CHI 2005, Portland. [Online] www-personal.si.umich.edu/ shakmatt/ CHI2005/ beyond_threaded_abstract. pdf (14.08.2006).

Rosenzweig, R. (2006): Can History be Open Source? Wikipedia and the Future of the Past. In: The Journal of American History, 93(1), 117-146.

Sanger, L. (2004): Why Wikipedia Must Jettison its Anti-Elitism. [Online] http://www.kuro5hin. org/story/2004/12/30/142458/25 (14.08.2006).

Sanger, L. (2005): The Early History of Nupedia and Wikipedia: A Memoir. [Online] http:// features.slashdot.org/article.pl?sid=05/04/18/164213\&tid=95\&tid=149\&tid=9 (14.08.2006).

Schliecker, C./K. Lehmann (2005): Verknüpft, Verknüpfter, Wikis. In: K. Lehmann /M. Schetsche (Hg.): Die Google-Gesellschaft. Vom digitalen Wandel des Wissens. Bielefeld: Transcript, $253-262$.

Schmidt, J. (2006): Weblogs. Eine kommunikationssoziologische Studie. Konstanz: UVK.

Schroer, J. et al. (2005): Wikipedia: Motivation für die freiwillige Mitarbeit an einer offenen Web- 
basierten Enzyklopädie. Poster beim 4. Treffen der Sektion „Arbeits-, Industrie- und Organisationspsychologie" der Deutschen Psychologischen Gesellschaft, Bonn.

Schroer, J./G. Hertel (2006): Das Phänomen Wikipedia: Warum arbeiten Personen unentgeltlich in Internet-basierten Projekten? Oder: Wikipedians, and Why They Do It. Vortrag beim 22. Symposium „Methoden der Sozialpsychologie“, Universität Hamburg.

Scotchmer, S. (2002): Local Public Goods and Clubs. In: A. J. Auerbach/M. Feldstein (Hg.): Handbook of Public Economics. Bd. 4. Amsterdam et al.: Elsevier, 1997-2042.

Seidenglanz, S./C. Pentzold (2006): Motivation Revisited. The Open Question of User Motivation in the Light of System Theory. Poster auf der Wikimania'06, Harvard Law School, Cambridge/MA, 6.-8.8.2006.

Shah, S. (2005): Productive Controversy. In: Proceedings of the Wikimania'05, Frankfurt a. M. Wikimania-Paper SS1. [Online] http://en.wikibooks.org/wiki/ Wikimania05/Presentation-SS1 (14.08.2006).

Shirky, C. (2003): A Group is its Own Worst Enemy. In: Proceedings of the ETech'03. [Online] http://www.shirky.com/writings/group_enemy.html (14.08.2006).

Shirky, C. (2003a): Social Software and the Politics of Groups. [Online] http://shirky.com/writings/group_politics.html (14.08.2006).

Strauss, A. L./J. M. Corbin (1996): Grounded Theory. Grundlagen qualitativer Sozialforschung. Weinheim: Psychologie Verlags Union.

Stvilia, B. et al. (2005): Assessing Information Quality of a Community-Based Encyclopedia. In: Proceedings of the International Conference on Information Quality ICIQ 2005, Cambridge/ MA, 442-454. [Online] www.isrl.uiuc.edu/ stvilia/papers/quantWiki.pdf (14.08.2006).

Stvilia, B. et al. (2005a): Information Quality Discussion in Wikipedia. In: Proceedings of the ICKM'05, Kuala Lumpur. [Online] www.isrl.uiuc.edu/ stvilia/papers/qualWiki.pdf (14.08.2006).

Tobacman, J. (2005): The motivation of WP contributors. In: Proceedings of the Wikimania'05, Frankfurt a. M. Wikimania-Paper JT1. [Online] http://en.wikibooks.org/wiki/Wikimania05/ Paper-JT1(14.08.2006).

Viégas, F./M. Wattenberg/K. Dave (2004): Studying Cooperation and Conflict Between Authors with History Flow Visualization. In: Proceedings of CHI 2004, Wien. [Online] http://portal. acm.org/citation.cfm?id=985765 (14.08.2006).

Voß, J./P. Danowski (2004): Bibliothek, Information und Dokumentation in der Wikipedia. In: Information Wissenschaft und Praxis, 55(8).

Voß, J. (2005): Measuring Wikipedia. In: Proceedings of the ISSI'05, Stockholm [Online] http:// eprints.rclis.org/archive/00003610/01/MeasuringWikipedia2005.pdf (14.08.2006).

Wales, J. (2004): Wikipedia Sociographics. In: Proceedings of the 21 $1^{\text {st }}$ Chaos Communication Congress. [Online] www.ccc.de/congress/2004/fahrplan/files/372-wikipedia-sociographics-slides. $\operatorname{pdf}(14.08 .2006)$.

Wenger, E. (1999): Communities of Practice. Learning, Meaning, and Identity. Cambridge et al.: Cambridge University Press.

Wikimedia: The Wikipedia Community (2006). [Online] meta.wikimedia.org/wiki/The_Wikipedia_Community (14.08.2006).

Wikipedia: Benutzer (2006). [Online] de.wikipedia.org/wiki/Wikipedia:Benutzer (14.08.2006).

Wikipedia: Download (2006). [Online] de.wikipedia.org/wiki/Wikipedia:Download (14.08.2006).

Wikipedia: Neutraler_Standpunkt (2006). [Online] de.wikipedia.org/wiki/Wikipedia:Neutraler_Standpunkt (14.08.2006).

Wikipedia: Portal (2006). [Online] de.wikipedia.org/wiki/Wikipedia:Portal (14.08.2006).

Wikipedia: Richtlinien (2006). [Online] de.wikipedia.org/wiki/Wikipedia:Richtlinien (14.08.2006).

Wikipedia: Soft_security (2006). [Online] en.wikipedia.org/wiki/Soft_security (14.08.2006).

Wikipedia: Special:Statistics (2006). [Online] en.wikipedia.org/wiki/Special:Statistics (14.08.2006).

Wikipedia: Spezial:Statistics (2006). [Online] de.wikipedia.org/wiki/Spezial:Statistics (14.08.2006). 
Wikipedia: Wikipedians (2006). [Online] en.wikipedia.org/wiki/Wikipedia:Wikipedians (14.08.2006).

Willke, H. (1993): Systemtheorie. Eine Einführung in die Grundprobleme der Theorie sozialer Systeme. Stuttgart: Fischer.

Wirth, W./A. Brändle (2006): Wikipedia. Diffusion, Nutzung und Kooperationsmotivation. In: Zeitschrift für Medienpsychologie, 18(2), 76-80.

Yates, J./W. J. Orlikowski (1992). Genres of Organizational Communication: A Structurational Approach to Studying Communication and Media. In: Academy of Management Review, $17(2), 299-326$.

YouTube.com [Online] youtube.com (12.01.2007).

Zachte, E. (2006): Wikipedia-Statistik. [Online] de.wikipedia.org/wiki/Wikipedia:Statistik (14.08.2006).

Zlatić, V. et al. (2006): Wikipedias: Collaborative Web-based Encyclopedias as Complex Networks. [Online] www.citebase.org/fulltext?format=application \%2Fpdf\&identifier=oai \%3AarXiv. org \%3Aphysics \%2F0602149 (14.08.2006). 